\title{
Periodic solutions of a class of
}

\section{nonautonomous second-order Hamiltonian systems with nonsmooth potentials}

\section{Yan Ning* and Tianqing An}

\section{"Correspondence:}

ningkegood@126.com

College of Science, Hohai University,

Nanjing, 210098, China

\begin{abstract}
This paper is concerned with nonautonomous second-order Hamiltonian systems with nondifferentiable potentials. By using the nonsmooth critical point theory for locally Lipschitz functionals, we obtain some new existence results for the periodic solutions.
\end{abstract}

Keywords: periodic solutions; second-order Hamiltonian systems; saddle point theorem; discontinuous nonlinearities; locally Lipschitz continuous

\section{Introduction and main results}

In this paper we consider the following second-order differential inclusions systems:

$$
\left\{\begin{array}{l}
\ddot{u}(t)+A(t) u(t) \in \partial F(t, u(t)) \quad \text { a.e. } t \in[0, T], \\
u(0)-u(T)=\dot{u}(0)-\dot{u}(T)=0,
\end{array}\right.
$$

where $T>0, A(t)$ is a continuous symmetric matrix of order $N$ and $F:[0, T] \times \mathbb{R}^{N} \rightarrow \mathbb{R}$ is locally Lipschitz continuous in $x$ and $\partial F(t, x)$ denotes the Clarke subdifferential of $F$ for $x$. There have been a lot of contributions on problem (1); see, for example, [1-3] and the references therein.

When $F(t, x)$ is continuously differentiable in $x$, problem (1) becomes the second-order Hamiltonian system

$$
\left\{\begin{array}{l}
\ddot{u}(t)+A(t) u(t)=\nabla F(t, u(t)) \quad \text { a.e. } t \in[0, T] \\
u(0)-u(T)=\dot{u}(0)-\dot{u}(T)=0 .
\end{array}\right.
$$

The smooth system (2) has also been studied in the past decades and many excellent results appeared; see $[4,5]$. In those works, the following assumption is necessary:

(A) $F(t, x)$ is measurable in $t$ for every $x \in \mathbb{R}^{N}$ and continuously differentiable in $x$ for a.e. $t \in[0, T]$, and there exist $a \in C\left(\mathbb{R}^{+}, \mathbb{R}^{+}\right), b \in L^{1}\left(0, T ; \mathbb{R}^{+}\right)$such that

$$
|F(t, x)| \leq a(|x|) b(t), \quad|\nabla F(t, x)| \leq a(|x|) b(t)
$$

for all $x \in \mathbb{R}^{N}$ and a.e. $t \in[0, T]$, where $\mathbb{R}^{+}$is the set of all nonnegative real number. 
Throughout this paper, we always suppose that $F:[0, T] \times \mathbb{R}^{N} \rightarrow \mathbb{R}$ satisfies the following assumption:

(A') $F(t, x)$ is integrable in $t$ over $[0, T]$ for each $x \in \mathbb{R}^{N}$ and locally Lipschitz continuous in $x$ for each $t \in[0, T]$.

Let $H_{T}^{1}$ be the usual Sobolev space with norm

$$
\|u\|=\left(\int_{0}^{T}|u(t)|^{2} d t+\int_{0}^{T}|\dot{u}(t)|^{2} d t\right)^{1 / 2} .
$$

Definition 1.1 We call $u \in H_{T}^{1}$ a weak solution of (1) if the following inequality holds:

$$
-\int_{0}^{T}(\dot{u}(t), \dot{v}(t)) d t+\int_{0}^{T}(A(t) u(t), v(t)) d t \leq \int_{0}^{T} F^{0}(t, u(t) ; v(t)) d t, \quad \forall v \in H_{T}^{1},
$$

where $F^{0}(t, x ; y)$ denotes the generalized directional derivative of $F$ at $x$ along the direction $y$.

The main results of this paper are as follows.

Theorem 1.2 Suppose $F(t, x)$ satisfies $\left(\mathrm{A}^{\prime}\right)$ and the following conditions:

(i $\left.\mathrm{i}_{1}\right)$ There exist $f, g \in L^{1}\left(0, T ; \mathbb{R}^{+}\right)$and $\alpha \in[0,1)$ such that for all $x \in \mathbb{R}^{N}$ and a.e. $t \in[0, T]$,

$$
\xi \in \partial F(t, x) \quad \Rightarrow \quad|\xi| \leq f(t)|x|^{\alpha}+g(t)
$$

(i $\left.i_{2}\right)$ There exists a $\gamma \in L^{1}\left(0, T ; \mathbb{R}^{+}\right)$such that for all $x \in \mathbb{R}^{N}$ and a.e. $t \in[0, T]$,

$$
\frac{F(t, x)}{|x|^{2 \alpha}} \leq \gamma(t)
$$

$\left(\mathrm{i}_{3}\right)$ There exists a subset $E$ of $[0, T]$ with meas $(E)>0$ such that for a.e. $t \in E$,

$$
\frac{F(t, x)}{|x|^{2 \alpha}} \rightarrow-\infty \quad \text { as }|x| \rightarrow \infty
$$

Then problem (1) possesses at least one weak solution.

Theorem 1.3 Suppose $F(t, x)$ satisfies $\left(\mathrm{A}^{\prime}\right),\left(\mathrm{i}_{1}\right)$ above and the following conditions:

$\left(\mathrm{i}_{2}^{\prime}\right)$ There exists $\gamma \in L^{1}\left(0, T ; \mathbb{R}^{+}\right)$such that

$$
\frac{F(t, x)}{|x|^{2 \alpha}} \geq-\gamma(t)
$$

for all $x \in \mathbb{R}^{N}$ and a.e. $t \in[0, T]$.

$\left(\mathrm{i}_{3}^{\prime}\right)$ There exists a subset $E$ of $[0, T]$ with meas $(E)>0$ such that

$$
\frac{F(t, x)}{|x|^{2 \alpha}} \rightarrow+\infty \quad \text { as }|x| \rightarrow \infty
$$

for a.e. $t \in E$.

Then problem (1) possesses at least one weak solution. 
The method in our paper is based on the nonsmooth least action principle and saddle point theorem initiated by Chang [6], different from [1] and [2] based on the nonsmooth mountain pass theorem. Moreover, we consider the case that the growth of the subdifferential $\partial F(t, x)$ in $x$ is sublinear $(\alpha \in[0,1))$, while in [1] and [2] the authors considered the case $\alpha \geq 1$.

Barletta and Papageorgiou [3, Theorem 3.4] proved the existence of solutions for problem (1), where they assumed that

- $F(t, x) \geq-\gamma(t), \forall x \in \mathbb{R}^{N}, t \in[0, T]$,

- $F(t, x) \rightarrow+\infty$ as $|x| \rightarrow \infty$ and $t \in E$,

- $\operatorname{dim}\left(\operatorname{span}\left\{u \in H_{T}^{1} \mid-\ddot{u}-A(t) u=\lambda u\right.\right.$ for some $\left.\left.\lambda<0\right\}\right)=0$.

By comparison, the first two assumptions are strengthened to $\left(i_{2}^{\prime}\right)$ and $\left(i_{3}^{\prime}\right)$, but the third one is not necessarily needed in the present paper.

Remark 1.4 If $F(t, x)$ is measurable in $t$ for every $x \in \mathbb{R}^{N}$ and continuously differentiable in $x$ for a.e. $t \in[0, T]$, the inequality (4) becomes

$$
|\nabla F(t, x)| \leq f(t)|x|^{\alpha}+g(t)
$$

Meanwhile, the above inequality (3) takes the form

$$
-\int_{0}^{T}(\dot{u}(t), \dot{v}(t)) d t+\int_{0}^{T}(A(t) u(t), v(t)) d t=\int_{0}^{T}(\nabla F(t, u(t)) ; v(t)) d t, \quad \forall v \in H_{T}^{1} .
$$

Then Theorem 1.2 and Theorem 1.3 generalize Theorem 2 and Theorem 3 of [5], respectively, without assuming the linear second-order system $\ddot{u}(t)+A(t) u(t)=0$ a.e. $t \in[0, T]$ has a nonzero solution, which is necessary in [5].

\section{Example 1.5 Let}

$$
F(t, x)=-|\sin \omega t|\left(|x|^{1+r}+|x|\right)
$$

for all $(t, x) \in[0, T] \times \mathbb{R}^{N}$, where $r \in[0,1), \omega=\frac{2 \pi}{T}$. Then $F$ satisfies the conditions of Theorem 1.2 , but it is not covered by the results of $[1,2,4,5]$.

\section{Basic definitions and preliminary results}

Let $(X,\|\cdot\|)$ be a real Banach space. We denote by $X^{*}$ the dual space of $X$, while $\langle\cdot, \cdot\rangle$ stands for the duality pairing between $X$ and $X^{*}$. A functional $h: X \rightarrow \mathbb{R}$ is called locally Lipschitz continuous if for every $u \in X$ there correspond a neighborhood $V_{u}$ of $u$ and a constant $L_{u} \geq 0$ such that

$$
|h(z)-h(w)| \leq L_{u}\|z-w\|, \quad \forall z, w \in V_{u} .
$$

If $u, v \in X$, we write $h^{0}(u ; v)$ for the generalized directional derivative of $h$ at the point $u$ along the direction $v$, i.e.,

$$
h^{0}(u ; v):=\limsup _{w \rightarrow u, t \rightarrow 0^{+}} \frac{h(w+t v)-h(w)}{t} .
$$

It is well known that $h^{0}$ is upper semicontinuous on $X \times X$ (see [7], Proposition 2.1.1). 
For locally Lipschitz continuous functionals $h_{1}, h_{2}: X \rightarrow \mathbb{R}$, we have

$$
\left(h_{1}+h_{2}\right)^{0}(x ; z) \leq h_{1}^{0}(x ; z)+h_{2}^{0}(x ; z), \quad \forall x, z \in X .
$$

The generalized gradient of the function $h$ in $u$, denoted by $\partial h(u)$, is the set defined by

$$
\partial h(u):=\left\{u^{*} \in X^{*}:\left\langle u^{*}, v\right\rangle \leq h^{0}(u ; v), \forall v \in X\right\} .
$$

Proposition 2.1.2 of [7] ensures that $\partial h(u)$ turns out nonempty, convex, weak* compact, thus the function $\lambda(x)=\min _{w \in \partial h(x)}\|w\|_{X^{*}}$ exists and is lower semicontinuous, i.e., $\liminf _{x \rightarrow x_{0}} \lambda(x) \geq \lambda\left(x_{0}\right)$.

If $f, g: X \rightarrow X$ be locally Lipschitz continuous, then

$$
\partial(f+g)(x) \subset \partial f(x)+\partial g(x)
$$

A point $u \in X$ is said to be a critical point of $h$ if

$$
h^{0}(u ; v) \geq 0, \quad \forall v \in X .
$$

We say the locally Lipschitz functional $h$ satisfies the nonsmooth (PS) condition if any sequence $\left\{x_{n}\right\}$ in $X$ such that $\left\{h\left(x_{n}\right)\right\}$ is bounded and $\lambda\left(x_{n}\right) \rightarrow 0$ possesses a convergent subsequence.

For more details, we can refer to [8-11]. To prove Theorem 1.2 and Theorem 1.3 in the next section, first we state the following well-known results.

Lemma 2.1 ([7], Theorem 2.3.7) Let $x$ and $y$ be points in X, and suppose that $f$ is Lipschitz on open set containing the line segment $[x, y]$. Then there exists a point $u$ in $(x, y)$ such that

$$
f(y)-f(x) \in\langle\partial f(u), y-x\rangle
$$

Lemma 2.2 ([6], Theorem 3.3) Let X be a real Banach space, and let $f$ be a locally Lipschitz function defined on $X$ satisfying the nonsmooth (PS) condition. Suppose $X=X_{1} \oplus X_{2}$ with a finite-dimensional subspace $X_{1}$, and there exist constants $b_{1}<b_{2}$ and a bounded neighborhood $N$ of $\theta$ in $X_{1}$ such that

$$
\left.f\right|_{X_{2}} \geq b_{2},\left.\quad f\right|_{\partial N} \leq b_{1} .
$$

Then $f$ has a critical point.

Lemma 2.3 ([12], Lemma 2) Suppose that $E$ is a measurable subset of $[0, T]$ and $G(t, x)$ is continuous in $x$ for a.e. $t \in E$. Assume that

$$
G(t, x) \rightarrow-\infty \quad \text { as }|x| \rightarrow \infty
$$

for a.e. $t \in E$. Then for every $\delta>0$ there exists a subset $E_{\delta}$ of $E$ with meas $\left(E / E_{\delta}\right)<\delta$ such that

$$
G(t, x) \rightarrow-\infty \text { as }|x| \rightarrow \infty
$$

uniformly for all $t \in E_{\delta}$. 
Lemma 2.4 ([6], Theorem 3.5) Suppose a locally Lipschitz functional $f$, defined on a reflexive Banach space X, satisfies the nonsmooth (PS) condition and is bounded from below. Then $c=\inf _{X} f(x)$ is a critical value of $f$.

\section{Proof of theorems}

For every $u \in H_{T}^{1}$, let $\bar{u}=\frac{1}{T} \int_{0}^{T} u(t) d t, \tilde{u}(t)=u(t)-\bar{u}$. Then the following inequalities hold:

$$
\begin{aligned}
& \|\tilde{u}\|_{\infty}^{2} \leq \frac{T}{12} \int_{0}^{T}|\dot{u}(t)|^{2} d t \quad \text { (Sobolev's inequality), } \\
& \int_{0}^{T}|\tilde{u}(t)|^{2} d t \leq \frac{T^{2}}{4 \pi^{2}} \int_{0}^{T}|\dot{u}(t)|^{2} d t \quad \text { (Wirtinger's inequality), } \\
& \|u\|_{\infty} \leq C\|u\|,
\end{aligned}
$$

where $C>0$ is a constant and $\|u\|_{\infty}=\max _{t \in[0, T]}|u(t)|$.

Define two functionals $\varphi: H_{T}^{1} \rightarrow \mathbb{R}$ and $\psi: H_{T}^{1} \rightarrow \mathbb{R}$ as follows:

$$
\begin{aligned}
& \varphi(u)=\frac{1}{2} \int_{0}^{T}|\dot{u}(t)|^{2} d t-\frac{1}{2} \int_{0}^{T}(A(t) u(t), u(t)) d t+\int_{0}^{T} F(t, u(t)) d t, \\
& \psi(u)=\int_{0}^{T} F(t, u(t)) d t .
\end{aligned}
$$

It is easy to verify that they are locally Lipschitz continuous on $H_{T}^{1}$, so it makes sense to consider their generalized directional derivatives $\varphi^{0}$ and $\psi^{0}$ :

$$
\varphi^{0}(u ; v)=\int_{0}^{T}(\dot{u}(t), \dot{v}(t)) d t-\int_{0}^{T}(A(t) u(t), v(t)) d t+\psi^{0}(u ; v), \quad \forall v \in H_{T}^{1} .
$$

Equation (9) at p.84 of [7] gives

$$
\psi^{0}(u ; v) \leq \int_{0}^{T} F^{0}(t, u(t) ; v(t)) d t, \quad \forall u, v \in H_{T}^{1} .
$$

Moreover, by [7], Theorem 2.7.5, one has

$$
\partial \psi(u) \subset \int_{0}^{T} \partial F(t, u(t)) d t
$$

i.e., to every $\xi \in \partial \psi(u)$ there corresponds a mapping $t \rightarrow q(t)$ from $[0, T]$ to $\left(H_{T}^{1}\right)^{*}$ with $q(t) \in \partial F(t, u(t))$ a.e. $t \in[0, T]$ such that for every $v \in H_{T}^{1}$,

$$
\langle\xi, v\rangle=\int_{0}^{T}(q(t), v(t)) d t .
$$

If $u \in H_{T}^{1}$ is a critical point of $\varphi$, i.e., $\theta \in \partial \varphi(u)$, then

$$
\begin{aligned}
& \int_{0}^{T}(\dot{u}(t), \dot{v}(t)) d t-\int_{0}^{T}(A(t) u(t), v(t)) d t+\int_{0}^{T} F^{0}(t, u(t) ; v(t)) d t \\
& \quad \geq \varphi^{0}(u ; v) \geq 0, \quad \forall v \in H_{T}^{1} .
\end{aligned}
$$

Thus, the critical points of $\varphi$ correspond to the solutions of problem (3). 
In the same time, there exists $q_{0}(t) \in \partial F(t, u)$ such that for all $v \in H_{T}^{1}$,

$$
0=\langle\theta, v\rangle=\int_{0}^{T}(\dot{u}(t), \dot{v}(t)) d t-\int_{0}^{T}(A(t) u(t), v(t)) d t+\int_{0}^{T}\left(q_{0}(t), v(t)\right) d t,
$$

it follows easily that $q_{0}(t)=\ddot{u}(t)$ a.e. $t \in[0, T]$, thus

$$
\ddot{u}(t)+A(t) u(t) \in \partial F(t, u(t)) \quad \text { a.e. on }[0, T],
$$

so that $u$ satisfies the system (1) too.

Define the subspaces of $H_{T}^{1}$ by

$$
\begin{aligned}
& W^{-} \triangleq \operatorname{span}\left\{u \in H_{T}^{1} \mid-\ddot{u}-A(t) u=\lambda u \text { for some } \lambda<0\right\}, \\
& V \triangleq \operatorname{span}\left\{u \in H_{T}^{1} \mid-\ddot{u}-A(t) u=0\right\}, \\
& W^{+} \triangleq \operatorname{span}\left\{u \in H_{T}^{1} \mid-\ddot{u}-A(t) u=\lambda u \text { for some } \lambda>0\right\} .
\end{aligned}
$$

It is easy to verify that $W^{-}$is finite-dimensional and there exists $\delta>0$ such that

$$
\begin{aligned}
& \int_{0}^{T}|\dot{u}(t)|^{2} d t-\int_{0}^{T}(A(t) u(t), u(t)) d t \geq \delta\|u\|^{2}, \quad \forall u \in W^{+}, \\
& \int_{0}^{T}|\dot{u}(t)|^{2} d t-\int_{0}^{T}(A(t) u(t), u(t)) d t \leq-\delta\|u\|^{2}, \quad \forall u \in W^{-} .
\end{aligned}
$$

Decompose $H_{T}^{1}$ as $H_{T}^{1}=W^{-} \oplus V \oplus W^{+}$, and denote $W=W^{+}+W^{-}$.

Lemma 3.1 Suppose that (5) and (6) hold. Assume that $u_{n}=v_{n}+w_{n}, v_{n} \in V, w_{n} \in W$ satisfying $\left\|u_{n}\right\| \rightarrow \infty(n \rightarrow \infty)$ and $\lim \sup _{n \rightarrow \infty}\left\|w_{n}\right\|\left\|<u_{n}\right\|^{\alpha}<+\infty$. Then

$$
\left\|u_{n}\right\|^{-2 \alpha} \int_{0}^{T} F\left(t, u_{n}(t)\right) d t \rightarrow-\infty \quad \text { as } n \rightarrow \infty
$$

Proof As the proof of Lemma 3 in [5], for every $\beta>0$, there exists $m_{\beta}>0$ such that

$$
\operatorname{meas}\left\{t \in(0, T)|| v(t) \mid<m_{\beta}\|v\|\right\}<\beta
$$

for all $v \in V$.

Let $B=\left\{t \in(0, T)|| v(t) \mid \geq m_{\beta}\|v\|\right\}$ for all $v \in V$, then we have meas $((0, T) \backslash B)<\beta$. By (6) and Lemma 2.3, there exists subset $E_{\delta}$ of $E$ with meas $\left(E \backslash E_{\delta}\right)<\delta$ such that

$$
\frac{F(t, x)}{|x|^{2 \alpha}} \rightarrow-\infty \quad \text { as }|x| \rightarrow \infty
$$

uniformly for all $t \in E_{\delta}$. Hence,

$$
\operatorname{meas}\left(B \cap E_{\delta}\right) \geq \operatorname{meas}\left(E_{\delta}\right)-\operatorname{meas}((0, T) \backslash B) \geq \operatorname{meas} E-\delta-\beta>0
$$

for $\delta$ and $\beta$ small enough. 
By (8), for every $\eta>0$, there exists an $M>0$ such that

$$
\frac{F(t, x)}{|x|^{2 \alpha}} \leq-\eta
$$

for all $|x| \geq M$ and a.e. $t \in E_{\delta}$. Furthermore, it follows from (5) that

$$
F(t, x) \leq-\eta|x|^{2 \alpha}+\gamma_{0}(t)
$$

for all $x \in \mathbb{R}^{N}$, a.e. $t \in E_{\delta}$ and some $\gamma_{0}(t)=M^{2 \alpha}(\eta+\gamma(t))$.

From (9) we obtain

$$
\int_{B \cap E_{\delta}} F(t, v) d t \leq-\eta m_{\beta}^{2 \alpha}\|v\|^{2 \alpha} \operatorname{meas}\left(B \cap E_{\delta}\right)+\int_{0}^{T} \gamma_{0}(t) d t .
$$

By (5) and (7) we have

$$
\int_{[0, T] \backslash\left(B \cap E_{\delta}\right)} F(t, v) d t \leq C^{2 \alpha}\|v\|^{2 \alpha} \int_{0}^{T} \gamma(t) d t .
$$

Since $\left\|u_{n}\right\| \rightarrow \infty(n \rightarrow \infty)$ and $\lim \sup _{n \rightarrow \infty} \frac{\left\|w_{n}\right\|}{\left\|u_{n}\right\|^{\alpha}}<+\infty$, by a simple computation we obtain $\left\|v_{n}\right\| \rightarrow \infty$ as $n \rightarrow \infty$, and $V \neq\{0\}$.

Hence, one has

$$
\limsup _{v \in V,\|v\| \rightarrow \infty}\|v\|^{-2 \alpha} \int_{0}^{T} F(t, v) d t \leq-\eta m_{\beta}^{2 \alpha} \operatorname{meas}\left(B \cap E_{\delta}\right)+C^{2 \alpha} \int_{0}^{T} \gamma(t) d t,
$$

which implies that

$$
\limsup _{n \rightarrow \infty}\left\|v_{n}\right\|^{-2 \alpha} \int_{0}^{T} F\left(t, v_{n}\right) d t \rightarrow-\infty
$$

by the arbitrariness of $\eta$.

By (4), (7), and Lemma 2.1, there exist $s \in[0,1]$ and $\xi_{n} \in \partial F\left(t, v_{n}+s w_{n}\right)$ such that

$$
\begin{aligned}
& \left|\int_{0}^{T} F\left(t, u_{n}\right) d t-\int_{0}^{T} F\left(t, v_{n}\right) d t\right| \\
& \quad=\left|\int_{0}^{T}\left\langle\xi_{n}, u_{n}-v_{n}\right\rangle d t\right| \leq \int_{0}^{T}\left|\xi_{n}\right|\left|w_{n}\right| d t \leq \int_{0}^{T}\left(f(t)\left|v_{n}+s w_{n}\right|^{\alpha}+g(t)\right)\left|w_{n}\right| d t \\
& \quad \leq \int_{0}^{T} f(t)\left(\left|v_{n}\right|^{\alpha}+\left|w_{n}\right|^{\alpha}\right)\left|w_{n}\right| d t+\int_{0}^{T} g(t)\left|w_{n}\right| d t \\
& \quad \leq\|f\|_{L^{1}}\left(\left\|v_{n}\right\|_{\infty}^{\alpha}+\left\|w_{n}\right\|_{\infty}^{\alpha}\right)\left\|w_{n}\right\|_{\infty}+\|g\|_{L^{1}}\left\|w_{n}\right\|_{\infty} \\
& \quad \leq C^{\alpha+1}\|f\|_{L^{1}}\left(\left\|v_{n}\right\|^{\alpha}+\left\|w_{n}\right\|^{\alpha}\right)\left\|w_{n}\right\|+C\|g\|_{L^{1}}\left\|w_{n}\right\|
\end{aligned}
$$

for all $n$. Since $\lim \sup _{n \rightarrow \infty} \frac{\left\|w_{n}\right\|}{\left\|u_{n}\right\|^{\alpha}}<+\infty$, one has

$$
C_{1} \triangleq \limsup _{n \rightarrow \infty}\left|\left\|u_{n}\right\|^{-2 \alpha} \int_{0}^{T} F\left(t, u_{n}\right) d t-\left\|u_{n}\right\|^{-2 \alpha} \int_{0}^{T} F\left(t, v_{n}\right) d t\right|<+\infty
$$


It follows from (10) that

$$
\begin{aligned}
\limsup _{n \rightarrow \infty}\left\|u_{n}\right\|^{-2 \alpha} \int_{0}^{T} F\left(t, u_{n}\right) d t & \leq \limsup _{n \rightarrow \infty}\left\|u_{n}\right\|^{-2 \alpha} \int_{0}^{T} F\left(t, v_{n}\right) d t+C_{1} \\
& \leq \limsup _{n \rightarrow \infty}\left\|v_{n}\right\|^{-2 \alpha} \int_{0}^{T} F\left(t, v_{n}\right) d t+C_{1}=-\infty
\end{aligned}
$$

Hence we obtain

$$
\left\|u_{n}\right\|^{-2 \alpha} \int_{0}^{T} F\left(t, u_{n}\right) d t \rightarrow-\infty
$$

as $n \rightarrow \infty$, which completes the proof.

Lemma 3.2 Under the conditions (4), (5) and (6), $\varphi$ satisfies the nonsmooth (PS) condition.

Proof Let $\left\{u_{n}\right\}$ be a sequence in $H_{T}^{1}$ such that $\left\{\varphi\left(u_{n}\right)\right\}$ is bounded and $\lambda\left(u_{n}\right)=$ $\min _{x^{*} \in \partial \varphi\left(u_{n}\right)}\left\|x^{*}\right\| \rightarrow 0$ as $n \rightarrow \infty$. Put $u_{n}^{*} \in \partial \varphi\left(u_{n}\right)$ such that $\left\|u_{n}^{*}\right\|=\lambda\left(u_{n}\right)=o(1)$, then there exists some integer $n_{0}$ such that for each $n \geq n_{0}$, we have

$$
\left|\left\langle u_{n}^{*}, h\right\rangle\right| \leq\|h\| \quad \text { for all } h \in H_{T}^{1} .
$$

Let $q_{n}(t) \in \partial\left(F\left(t, u_{n}(t)\right)\right)$ such that

$$
\left\langle u_{n}^{*}, h\right\rangle=\int_{0}^{T}\left(\dot{u}_{n}(t), \dot{h}(t)\right) d t-\int_{0}^{T}\left(A(t) u_{n}(t), h(t)\right) d t+\int_{0}^{T}\left(q_{n}(t), h(t)\right) d t, \quad \forall h \in H_{T}^{1}
$$

Firstly, we show that $\left\{u_{n}\right\}$ is bounded. If $\left\{u_{n}\right\}$ is unbounded, without loss of generality we may assume that $\left\|u_{n}\right\| \rightarrow \infty$ as $n \rightarrow \infty$. Split $u_{n}=v_{n}+w_{n}=v_{n}+w_{n}^{+}+w_{n}^{-} \subset V \oplus W^{+} \oplus W^{-}$. It follows from (4) and (7) that

$$
\begin{aligned}
\left|\int_{0}^{T}\left\langle q_{n}(t), w_{n}^{+}\right\rangle d t\right| & \leq\|f\|_{L^{1}}\left\|u_{n}\right\|_{\infty}^{\alpha}\left\|w_{n}^{+}\right\|_{\infty}+\|g\|_{L^{1}}\left\|w_{n}^{+}\right\|_{\infty} \\
& \leq C^{\alpha+1}\|f\|_{L^{1}}\left\|u_{n}\right\|^{\alpha}\left\|w_{n}^{+}\right\|+C\|g\|_{L^{1}}\left\|w_{n}^{+}\right\|
\end{aligned}
$$

for all $n$. Hence,

$$
\begin{aligned}
\left\|w_{n}^{+}\right\| & \geq\left\|u_{n}^{*}\right\|\left\|w_{n}^{+}\right\| \geq\left\langle u_{n}^{*}, w_{n}^{+}\right\rangle \\
& =\int_{0}^{T}\left|\dot{w}_{n}^{+}\right|^{2} d t-\int_{0}^{T}\left(A(t) w_{n}^{+}, w_{n}^{+}\right) d t+\int_{0}^{T}\left(q_{n}(t), w_{n}^{+}(t)\right) d t \\
& \geq \delta\left\|w_{n}^{+}\right\|^{2}-C^{\alpha+1}\|f\|_{L^{1}}\left\|u_{n}\right\|^{\alpha}\left\|w_{n}^{+}\right\|-C\|g\|_{L^{1}}\left\|w_{n}^{+}\right\|,
\end{aligned}
$$

which implies that $\lim \sup _{n \rightarrow \infty} \frac{\left\|w_{n}^{+}\right\|}{\left\|u_{n}\right\|^{\alpha}}<+\infty$. In a similar way

$$
\left|\int_{0}^{T}\left(q_{n}(t), w_{n}^{-}(t)\right) d t\right| \leq C^{\alpha+1}\|f\|_{L^{1}}\left\|u_{n}\right\|^{\alpha}\left\|w_{n}^{-}\right\|+C\|g\|_{L^{1}}\left\|w_{n}^{-}\right\|
$$


for all $n$. Thus one obtains

$$
\begin{aligned}
-\left\|u_{n}^{*}\right\|\left\|w_{n}^{-}\right\| & \leq\left\langle u_{n}^{*}, w_{n}^{-}\right\rangle \\
& =\int_{0}^{T}\left|\dot{w}_{n}^{-}\right|^{2} d t-\int_{0}^{T}\left(A(t) w_{n}^{-}, w_{n}^{-}\right) d t+\int_{0}^{T}\left(q_{n}(t), w_{n}^{-}(t)\right) d t \\
& \leq-\delta\left\|w_{n}^{-}\right\|^{2}+C^{\alpha+1}\|f\|_{L^{1}}\left\|u_{n}\right\|^{\alpha}\left\|w_{n}^{-}\right\|+C\|g\|_{L^{1}}\left\|w_{n}^{-}\right\| .
\end{aligned}
$$

This means $\lim \sup _{n \rightarrow \infty} \frac{\left\|w_{n}^{-}\right\|}{\left\|u_{n}\right\|^{\alpha}}<+\infty$. Hence we have

$$
\limsup _{n \rightarrow \infty} \frac{\left\|w_{n}\right\|}{\left\|u_{n}\right\|^{\alpha}}<+\infty
$$

By the boundedness of $\varphi\left(u_{n}\right)$ and the continuity of $A(\cdot)$, there exists a constant $C_{2} \geq 1$ such that

$$
\begin{aligned}
\int_{0}^{T} F\left(t, u_{n}\right) d t & =\varphi\left(u_{n}\right)-\frac{1}{2} \int_{0}^{T}\left|\dot{w}_{n}\right|^{2} d t+\frac{1}{2} \int_{0}^{T}\left(A(t) w_{n}, w_{n}\right) d t \\
& \geq-C_{2}-\frac{1}{2} \int_{0}^{T}\left|\dot{w}_{n}\right|^{2} d t-\frac{1}{2} C_{2} \int_{0}^{T}\left|w_{n}\right|^{2} d t \\
& \geq-C_{2}-\frac{1}{2} C_{2}\left\|w_{n}\right\|^{2}
\end{aligned}
$$

for all $n$. Furthermore, it follows from (12) that

$$
\liminf _{n \rightarrow \infty}\left\|u_{n}\right\|^{-2 \alpha} \int_{0}^{T} F\left(t, u_{n}\right) d t>-\infty
$$

which contradicts Lemma 3.1. Hence $\left\{u_{n}\right\}$ is bounded in $H_{T}^{1}$, thus there exists an $u \in H_{T}^{1}$ such that $u_{n} \rightarrow u$ in $H_{T}^{1}$ and $u_{n} \rightarrow u$ in $C\left([0, T], \mathbb{R}^{N}\right)$, where a subsequence is considered when necessary.

Since $H_{T}^{1}$ is reflexive while $\partial \varphi(u)$ is weak* compact, and the set-valued mapping $u \rightarrow$ $\partial \varphi(u)$ is upper semicontinuous, we can find an $u^{*} \in \partial \varphi(u)$ such that

$$
\left\langle u_{n}^{*}-u^{*}, u_{n}-u\right\rangle \rightarrow 0 \quad \text { as } n \rightarrow \infty
$$

On the other hand,

$$
\begin{aligned}
\left\langle u_{n}^{*}-u^{*}, u_{n}-u\right\rangle= & \int_{0}^{T}\left|\dot{u}_{n}(t)-\dot{u}(t)\right|^{2} d t \\
& -\int_{0}^{T}\left(A(t)\left(u_{n}(t)-u(t)\right), u_{n}(t)-u(t)\right) d t \\
& +\int_{0}^{T}\left(q_{n}(t)-q(t), u_{n}(t)-u(t)\right) d t,
\end{aligned}
$$

where $q_{n}(t) \in \partial F\left(t, u_{n}(t)\right)$ and $q(t) \in \partial F(t, u(t))$. From a simple computation we obtain $\int_{0}^{T}\left|\dot{u}_{n}-\dot{u}\right|^{2} d t \rightarrow 0$ as $n \rightarrow \infty$, and hence $u_{n} \rightarrow u$ in $H_{T}^{1}$. Therefore, $\varphi$ satisfies the nonsmooth (PS) condition. 
Proof of Theorem 1.2 We verify that $\varphi$ satisfies the other conditions of Lemma 2.2. Firstly we prove that

$$
\varphi(u) \rightarrow+\infty \quad \text { as }\|u\| \rightarrow \infty \text { in } W^{+} .
$$

By (4), (7), and Lemma 2.1, there exist $s \in[0,1]$ and $\xi \in \partial F(t, s u)$ such that

$$
\begin{aligned}
& \left|\int_{0}^{T} F(t, u) d t-\int_{0}^{T} F(t, 0) d t\right| \\
& \quad=\left|\int_{0}^{T}\langle\xi, u\rangle d t\right| \leq\|f\|_{L^{1}}\|u\|_{\infty}^{\alpha}\|u\|_{\infty}+\|g\|_{L^{1}}\|u\|_{\infty} \\
& \quad \leq C^{\alpha+1}\|f\|_{L^{1}}\|u\|^{\alpha+1}+C\|g\|_{L^{1}}\|u\|
\end{aligned}
$$

for all $n$. Hence we obtain

$$
\begin{aligned}
\varphi(u)= & \frac{1}{2} \int_{0}^{T}|\dot{u}(t)|^{2} d t-\frac{1}{2} \int_{0}^{T}(A(t) u(t), u(t)) d t+\int_{0}^{T} F(t, u(t)) d t \\
\geq & \frac{1}{2} \int_{0}^{T}|\dot{u}(t)|^{2} d t-\frac{1}{2} \int_{0}^{T}(A(t) u(t), u(t)) d t+\int_{0}^{T} F(t, 0) d t \\
& -\left|\int_{0}^{T} F(t, u(t)) d t-\int_{0}^{T} F(t, 0) d t\right| \\
\geq & \frac{1}{2} \delta\|u\|^{2}-C^{\alpha+1}\|f\|_{L^{1}}\|u\|^{\alpha+1}-C\|g\|_{L^{1}}\|u\|+\int_{0}^{T} F(t, 0) d t
\end{aligned}
$$

for all $u \in W^{+}$. Since $\alpha \in[0,1)$, it is clear that (13) holds.

Secondly we show that

$$
\varphi(u) \rightarrow-\infty \quad \text { as }\|u\| \rightarrow \infty \text { in } W^{-} \oplus V .
$$

Arguing by contradiction, assume that there exist $M \in \mathbb{R}$ and a sequence $\left\{u_{n}\right\} \subset W^{-} \oplus V$ such that $\left\|u_{n}\right\| \rightarrow \infty$ as $n \rightarrow \infty$ and

$$
\varphi\left(u_{n}\right) \geq M
$$

for all $n$. Write $u_{n}=v_{n}+w_{n}^{-}, v_{n} \in V, w_{n}^{-} \in W^{-}$. We consider the case that $\left\{u_{n}\right\}$ has a subsequence, say $\left\{u_{n}\right\}$, such that $\lim \sup _{n \rightarrow \infty} \frac{\left\|w_{n}^{-}\right\|}{\left\|u_{n}\right\|^{\alpha}}<+\infty$. By Lemma 3.1, one has

$$
\limsup _{n \rightarrow \infty}\left\|u_{n}\right\|^{-2 \alpha} \int_{0}^{T} F\left(t, u_{n}\right) d t=-\infty
$$

Hence

$$
\limsup _{n \rightarrow \infty}\left\|u_{n}\right\|^{-2 \alpha} \varphi\left(u_{n}\right) \leq \limsup _{n \rightarrow \infty}\left\|u_{n}\right\|^{-2 \alpha} \int_{0}^{T} F\left(t, u_{n}\right) d t=-\infty,
$$

which contradicts (15). 
Now we consider the case that $\frac{\left\|w_{n}^{-}\right\|}{\left\|u_{n}\right\|^{\alpha}} \rightarrow+\infty$ as $n \rightarrow \infty$, in this case, $W^{-} \neq\{0\}$ and $\left\|w_{n}^{-}\right\| \rightarrow \infty$ as $n \rightarrow \infty$. From (11), one obtains that

$$
\begin{aligned}
\varphi(u)= & \frac{1}{2} \int_{0}^{T}\left|\dot{w}_{n}^{-}\right|^{2} d t-\frac{1}{2} \int_{0}^{T}\left(A(t) w_{n}^{-}, w_{n}^{-}\right) d t+\int_{0}^{T} F\left(t, u_{n}\right) d t \\
\leq & -\frac{1}{2} \delta\left\|w_{n}^{-}\right\|^{2}+\int_{0}^{T} F\left(t, v_{n}\right) d t+\left|\int_{0}^{T} F\left(t, u_{n}\right) d t-\int_{0}^{T} F\left(t, v_{n}\right) d t\right| \\
\leq & -\frac{1}{2} \delta\left\|w_{n}^{-}\right\|^{2}+C^{\alpha+1}\|f\|_{L^{1}}\left(\left\|v_{n}\right\|^{\alpha}+\left\|w_{n}^{-}\right\|^{\alpha}\right)\left\|w_{n}^{-}\right\| \\
& +C\|g\|_{L^{1}}\left\|w_{n}^{-}\right\|+\int_{0}^{T} F\left(t, v_{n}\right) d t
\end{aligned}
$$

for all $n$. It follows from (5) and (7) that

$$
\int_{0}^{T} F\left(t, v_{n}\right) d t \leq C^{2 \alpha}\left\|v_{n}\right\|^{2 \alpha} \int_{0}^{T} \gamma(t) d t \leq C^{2 \alpha}\left\|u_{n}\right\|^{2 \alpha} \int_{0}^{T} \gamma(t) d t
$$

which implies that

$$
\limsup _{n \rightarrow \infty}\left\|u_{n}\right\|^{-\alpha}\left\|w_{n}^{-}\right\|^{-1} \int_{0}^{T} F\left(t, v_{n}\right) d t \leq \limsup _{n \rightarrow \infty} C^{2 \alpha} \frac{\left\|u_{n}\right\|^{\alpha}}{\left\|w_{n}^{-}\right\|} \int_{0}^{T} \gamma(t) d t=0
$$

hence we obtain

$$
\limsup _{n \rightarrow \infty}\left\|u_{n}\right\|^{-\alpha}\left\|w_{n}^{-}\right\|^{-1} \varphi\left(u_{n}\right) \leq-\frac{1}{2} \delta \liminf _{n \rightarrow \infty} \frac{\left\|w_{n}^{-}\right\|}{\left\|u_{n}\right\|^{\alpha}}+2 C^{2 \alpha+1}\|f\|_{L^{1}}=-\infty
$$

which contradicts (15) too.

By Lemma 2.2, $\varphi(u)$ has a critical point $u \in H_{T}^{1}$. The proof is completed.

If $W^{-} \oplus V=\{0\}$, the solution of system (1) can be obtained according to Lemma 2.4.

Proof of Theorem 1.3 Similar to the proof of Lemma 3.2 and Theorem 1.2, we can prove that $\varphi$ satisfies the nonsmooth (PS) condition, and

$$
\varphi(u) \rightarrow+\infty \quad \text { as }\|u\| \rightarrow \infty \text { in } W^{+} \oplus V .
$$

If $W^{-}=\{0\}, \varphi$ has a minimum by Lemma 2.4. In the case of $W^{-} \neq\{0\}$, we have

$$
\varphi(u) \rightarrow-\infty \quad \text { as }\|u\| \rightarrow \infty \text { in } W^{-} .
$$

According to Lemma 2.2, $\varphi$ has a critical point.

The authors declare that they have no competing interests. 


\section{Acknowledgements}

The authors would like to express their heartfelt appreciations to the expert referees for their helpful comments and suggestions. The work was supported by the Fundamental Research Funds for the Central Universities (No. 2014B38214).

Received: 26 June 2014 Accepted: 23 January 2015 Published online: 20 February 2015

\section{References}

1. Papageorgiou, EH, Papageorgiou, NS: Nonlinear second order periodic systems with nonsmooth potential. Czechoslov. Math. J. 15, 347-371 (2004)

2. Denkowski, Z, Gasinski, L, Papageorgiou, NS: Multiple solutions for nonautonomous second order periodic systems. Acta Math. Sci. Ser. B Engl. Ed. 30(1), 350-358 (2010)

3. Barletta, G, Papageorgiou, NS: Nonautonomous second order periodic systems: existence and multiplicity of solutions. J. Nonlinear Convex Anal. 8(3), 373-390 (2007)

4. Mawhin, J, Willem, M: Critical Point Theory and Hamiltonian Systems. Springer, New York (1989)

5. Tang, CL, Wu, XP: Periodic solutions for a class of nonautonomous subquadratic second order Hamiltonian systems. J. Math. Anal. Appl. 275, 870-882 (2002)

6. Chang, KC: Variational methods for non-differentiable functionals and their applications to partial differential equations. J. Math. Anal. Appl. 80, 102-129 (1981)

7. Clarke, FH: Optimization and Nonsmooth Analysis. Classics Appl. Math., vol. 5. SIAM, Philadelphia (1990)

8. Szulkin, A: Minimax principles for lower semicontinuous functions and applications to nonlinear boundary value problems. Ann. Inst. Henri Poincaré, Anal. Non Linéaire 3, 77-109 (1986)

9. Panagiotopoulos, PD: Hemivariational Inequalities. Applications in Mechanics and Engineering. Springer, Berlin (1993)

10. Filippakis, ME: Existence and multiplicity results for nonlinear nonautonomous second-order systems. Nonlinear Anal. 68, 1611-1626 (2008)

11. Barletta, G, Marano, SA: Some remarks on critical point theory for locally Lipschitz functions. Glasg. Math. J. 45, 131-141 (2003)

12. Tang, $\mathrm{CL}, \mathrm{Wu}, \mathrm{XP}$ : Periodic solutions for second order Hamiltonian systems with not uniformly coercive potential. J. Math. Anal. Appl. 259, 386-397 (2001)

\section{Submit your manuscript to a SpringerOpen ${ }^{\ominus}$ journal and benefit from:}

- Convenient online submission

- Rigorous peer review

- Immediate publication on acceptance

- Open access: articles freely available online

- High visibility within the field

- Retaining the copyright to your article 